\title{
Foreword to the special issue on Bone and cartilage mechanobiology across the scales
}

\author{
Peter Pivonka $^{1}$ • J. W. Fernandez ${ }^{2}$ - P. J. Hunter ${ }^{3}$
}

Published online: 25 January 2016

(C) Springer-Verlag Berlin Heidelberg 2016

The idea for this special issue on "Bone and Cartilage Mechanobiology Across the Scales" in the journal Biomechanics and Modeling in Mechanobiology is based on a series of high-quality presentations at two minisymposia at the 11th World Congress on Computational Mechanics (WCCM, July 20-25, 2014, Barcelona, Spain) and at the 7th World Congress of Biomechanics (WCB, July 611, 2014, Boston, USA). A total of 13 papers have been selected to present an unprecedented fashion of current stateof-the-art developments in computational and experimental assessment of bone and cartilage including the mechanobiology of structural adaptation as well as the cellular and biochemical underpinnings of tissue remodeling. The two minisymposia which papers have been selected from are as follows: Bone and Cartilage Mechanobiology: Experimental and Computational Assessment Across the Scales (WCCM 2014) and Multiscale Techniques in Biomechanics and Mechanobiology (WCB 2014).

As a discipline at the interface of biology and engineering, mechanobiology has grown significantly in the past decade. Major efforts have been made to better characterize the effects of physical forces on cells, tissues and organs, while biologists, for a long time, have utilized a reductionist approach to study cell behavior where the major focus has been on how biochemical factors (i.e., hormones,

Peter Pivonka

peter.pivonka@unimelb.edu.au

1 St Vincent's Department of Surgery, University of Melbourne, Melbourne, VIC, Australia

2 Department of Engineering Science, Auckland Bioengineering Institute, University of Auckland, Auckland, New Zealand

3 Auckland Bioengineering Institute, University of Auckland, Auckland, New Zealand cytokines and drugs) modulate cell behavior. This paradigm has now shifted significantly toward a more holistic approach where the entire cell microenvironment is taken into account. It is now well accepted that mechanical forces are involved in development, physiology and disease. In particular, musculoskeletal (MSK) tissues and cells are regulated by mechanical loading, and this regulation is central to tissue homeostasis, tissue adaptation and disease processes such as osteoporosis, osteoarthritis and sarcopenia. Furthermore, the process of aging has been linked to changes in mechanotransduction, which affects several selected physiological systems, including the musculoskeletal and the cardiovascular systems. Latest therapeutic treatment strategies not only utilize pharmacological agents to target specific MSK tissues, but also prescribe patient-specific physical exercise regimes.

A common feature of these tissues is that they contain mechanosensory cells which sense a particular mechanical stimulus and translate it into a biochemical response. In bone, cartilage, tendon and muscles, osteocytes, chondrocytes, tenocytes and myocytes are thought to be the respective mechanosensory cells. A common principle of these tissues is their ability to adapt to changes in the mechanical loading environment by modifying tissue and organ scale material properties. In diseased states, this adaptation process is disrupted, leading to tissue degradation, loss of tissue quality, and ultimately to diseases such as osteoporosis, osteoarthritis and sarcopenia. Currently, the cellular mechanosensing mechanisms are still poorly understood. A lack of mechanistic understanding of the type of mechanical stimuli at different scales is one of the primary foci of mechanobiology, which, as a consequence, enjoys enormous potential to provide critical new insights into both physiological function and etiology of disease.

In this spirit, we open our collection of papers dealing with bone adaptation studies which in recent years has been a 
widely studied phenomenon. A major aspect of bone remodeling and adaptation is the fact that bone formation and bone resorption responses may be linked to different mechanical quantities such as hydrostatic pressure, strain-energy density, shear stress and others which can give rise to various adaptation patterns.

We start this series of papers with a multiscale poromicromechanical model of bone (Scheiner et al. 2015), which explores the effects of physiological bone strains on inducing hydrostatic pressure both in the vascular porosity and in the lacunar porosity. It is shown that physiological loading induces osteocyte stimulating lacunar pressure. Subsequently, a mechanostatistical model of three-dimensional cortical bone remodeling is presented and applied to in vivo equine data (Wang et al. 2015). This model uses the von Mises stress as a mechanobiological regulatory quantity to control cortical remodeling. The third paper on bone adaptation presents a comprehensive multiscale mechanobiological model of bone remodeling which includes hormonal regulation and biochemical coupling of bone cell populations, the influence of the microstructure on bone turnover rate, and mechanical adaptation of the tissue (Lerebours et al. 2015). This model is able to predict site-specific bone loss in the femur during osteoporosis and mechanical disuse. The fourth paper on bone remodeling by Villette and Phillips (2015) deals with phenomenological structural bone remodeling using a mechanistic poroelastic model. Goal of their study presented here was the isolation of phenomenological drivers based on the results of a mechanistic approach. The last paper on bone adaptation deals with large-scale simulations for predicting microstructural changes in whole human vertebrae due to mechanical loading (Badilatti et al. 2015). Ultimate aim of this work is to identify individuals at risk of bone fractures, which remains challenging despite recent advances in bone strength assessment.

The second series of papers deals with improving our current understanding of the mechanical properties of bone at different scales using both experimental and theoretical approaches. Along this line, the first paper deals with characterizing the anisotropic elastic properties of cortical bone at the human tibia mid-diaphysis and to elucidate their relationships with mass density (Bernard et al. 2015). Resonant ultrasound spectroscopy was used to measure the transverse isotropic stiffness tensor. The second paper on this topic presents a stochastic multiscale model of cortical bone elasticity based on high-resolution imaging (Sansalone et al. 2015). This approach allows accurate and reliable assessment of bone quality and bone mechanical properties. Subsequently, we present a novel analytical multiscale model for the collagen fibril which investigates molecular and intermolecular effects in collagen fibril mechanics (Marino 2015). A typical three-region shape and hysteresis behavior of a fibril-based constitutive response as well as the transition from a yielding-like to a brittle-like behavior are recovered with a special insight on the underlying nanoscale mechanisms. The last paper in this series examines the microstructure and molecular composition of different anatomical positions in the diaphysis of an osteogenesis imperfecta human tibia (Katti et al. 2015). The study shows that although there is no significant microstructural difference, molecular changes are observed using FTIR, revealing differences in molecular composition of the four anatomical positions.

The third series of papers deals with orthopedic and tissue engineering applications which become more and more important given the increasing occurrence of joint replacement demanding novel quantitative strategies for tissue regeneration. The first paper in this series deals with the development of a computational modeling tool, which allows to better understand and control the growth process of in vitro cultured neotissues toward obtaining functional tissues (Guyot et al. 2015). The second paper on orthopedics deals with finite-element simulations of the bone remodeling process of a femur implanted with a cementless total hip replacement and a hip resurfacing implant (Dickinson 2015). This study supports the application of predictive bone remodeling algorithms as one element in the range of physical and computational studies, which should be conducted in the preclinical evaluation of new prostheses.

Finally, our selection of papers for this special issue concludes with manuscripts dealing with cartilage mechanobiology. The first paper presents an evaluation of the role for cartilage fiber 'split line' orientation in informing femoral cartilage stress patterns (Shim et al. 2015). This model indicated that 'split-lines' are formed along the line of maximum principal strains and may have a biomechanical role of protecting the cartilage by limiting the cartilage deformation to the area of higher cartilage thickness. The second paper developed a method that can measure natural alterations in cartilage material properties in a murine joint (Berteau et al. 2015). Accurate and reliable experimental methods are a prerequisite for understanding changes in mechanical tissue properties during growth and are a critical step in advancing strategies for orthopedics and for cell and biomaterial-based therapies dedicated to cartilage repair.

Last but not least, we thank the editors of Biomechanics and Modeling in Mechanobiology for hosting this special issue, especially Gerhard A. Holzapfel for his invaluable help and guidance. Also we would like to express our thanks to Priya Balamurugan for her help in managing manuscripts and making sure deadlines are met. Finally, we hope that this selection of papers is a source of future inspiration. In particular, that biologists, orthopedic surgeons and clinicians can appreciate the strong potential that engineering sciences have to offer in exploring the role of mechanical forces in modulating biological responses. 


\section{References}

\section{Bone adaptation}

Badilatti SD, Christen P, Levchuk A, Marangalou JH, van Rietbergen B, Parkinson I, Müller R (2015) Large-scale microstructural simulation of load-adaptive bone remodeling in whole human vertebrae. Biomech Model Mechanobiol. doi:10.1007/s10237-015-0715-8

Lerebours Ch, Buenzli P, Scheiner S, Pivonka P (2015) A multiscale mechanobiological model of bone remodelling predicts site-specific bone loss in the femur during osteoporosis and mechanical disuse. Biomech Model Mechanobiol. doi:10.1007/ s10237-015-0705-x

Scheiner S, Pivonka P, Hellmich Ch (2015) Poromicromechanics reveals that physiological bone strains induce osteocyte stimulating lacunar pressure. Biomech Model Mechanobiol. doi:10.1007/ s10237-015-0704-y

Villette CC, Phillips ATM (2015) Informing phenomenological structural bone remodelling with a mechanistic poroelastic model. Biomech Model Mechanobiol. doi:10.1007/s10237-015-0735-4

Wang X, Thomas CDL, Clement JG, Das R, Davies H, Fernandez JW (2015) A mechanostatistical approach to cortical bone remodelling: an equine model. Biomech Model Mechanobiol. doi:10. 1007/s10237-015-0669-x

\section{Bone mechanical properties}

Bernard S, Schneider J, Varga P, Laugier P, Raum K, Grimal Q (2015) Elasticity-density and viscoelasticity-density relationships at the tibia mid-diaphysis assessed from resonant ultrasound spectroscopy measurements. Biomech Model Mechanobiol. doi:10. 1007/s10237-015-0689-6

Katti KS, Gu Ch, Katti DR (2015) Anisotropic properties of human cortical bone with osteogenesis imperfecta. Biomech Model Mechanobiol. doi:10.1007/s10237-015-0727-4

Marino M (2015) Molecular and intermolecular effects in collagen fibril mechanics: a multiscale analytical model compared with atomistic and experimental studies. Biomech Model Mechanobiol. doi:10. 1007/s10237-015-0707-8

Sansalone V, Gagliardi D, Desceliers Ch, Bousson V, Laredo J-D, Peyrin F, Haiat G, Naili S (2015) Stochastic multiscale modelling of cortical bone elasticity based on high-resolution imaging. Biomech Model Mechanobiol. doi:10.1007/s10237-015-0695-8
Orthopaedic and tissue engineering applications

Dickinson AS (2015) Activity intensity, assistive devices and joint replacement influence predicted remodelling in the proximal femur. Biomech Model Mechanobiol. doi:10.1007/ s10237-015-0678-9

Guyot Y, Papantoniou I, Luyten FP, Geris L (2015) Coupling curvature-dependent and shear stress stimulated neotissue growth in dynamic bioreactor cultures: a 3D computational model of a complete scaffold. Biomech Model Mechanobiol. doi:10.1007/ s10237-015-0753-2

\section{Cartilage mechanics}

Berteau J-Ph, Oyen M, Shefelbine SJ (2015) Permeability and shear modulus of articular cartilage in growing mice. Biomech Model Mechanobiol. doi:10.1007/s10237-015-0671-3

Shim VB, Besier TF, Lloyd DG, Mithraratne K, Fernandez JW (2015) The influence and biomechanical role of cartilage splitline pattern on tibiofemoral cartilage stress distribution during the stance phase of gait. Biomech Model Mechanobiol. doi:10.1007/ s10237-015-0668-y 